\title{
Itinerary Aware Data Delivery Technique for Underwater Acoustic Sensor Networks
}

\author{
V. Bharathi, P. Raja, S. Meenabavyakarthika, V. Logisvary
}

\begin{abstract}
Monitoring and maintaining aquatic environment is the universal need and Underwater Acoustic Sensor Networks (UASN) is an emerging technology plays a major role in acoustic data acquistion. The data acquisition is challenging issue in UASN due to its communication characteristics. Though, there are several geo-opportunistic routing protocols were explored to improve the data acquisition it can be still improved by enhanced routing technique. The existing Geo-graphical depth adjustment routing (GEDAR) uses Global Positioning System(GPS) based notes for improving data acquisition, however it consumes more energy and increases overhead. We make an attempt to study about efficient data acquisition process and its path reliability. The proposed Itinerary aware routing protocol(IARP) acquires neighboring node's information for constructing efficient and reliable link with minimum information which improves data delivery ratio with minimum energy consumption. The proposed IARP increases $11 \%$ packet delivery ratio and reduces delay by $13 \%$, and energy consumption by $9 \%$ comparing with existing GEDAR based algorithm. IARP also performs better than Depth based routing (DBR).
\end{abstract}

Keywords: Underwater Acoustic Sensor Networks, GEDAR, Depth based routing, Itinerary aware routing

\section{INTRODUCTION}

Around $66 \%$ of earth surface are represented by Oceans. Global production, absorption of carbon dioxide, climatical changes are mostly depends upon these ocean environments. Under water sensor network (UWSN) is a network of autonomous sensor nodes used to sense water quality, water temperature, and water pressure etc. The data sensed by each sensor is aggregated. Sonobuoys are the nodes placed on surface of the water which is utilized for collection of data from nodes placed under water and retransmit it to the central unit. Few characteristics of UWSN is similar to terrestrial sensor network, however UWSN uses acoustic channels instead of radio channels for communication between the nodes placed under water. These acoustic channels can support only lower bandwidth, huge propagation delay and more probability of error comparing with radio channels. Hence efficient UWSN is designed with large number of deployed nodes which can handle limited energy, less data storage, high probability of error and more latency.

Revised Manuscript Received on December 30, 2019.

* Correspondence Author

Dr. V. Bharathi, Professor, Department of ECE, Sri Manakula Vinayagar Engineering college ,Puducherry E-mail: bharathime@rediffmail.com

Dr. P. Raja, Professor, Department of ECE, Sri Manakula Vinayagar Engineering College, Puducherry E-mail: rajashruthy@gmail.com

S. Meenabavyakarthika, PG Student, Department of ECE, Sri Manakula Vinayagar Engineering college ,Puducherry

V. Logisvary, Assistant Professor, Department of ECE Sri Manakula Vinayagar Engineering College, Puducherry

(c) The Authors. Published by Blue Eyes Intelligence Engineering and Sciences Publication (BEIESP). This is an open access article under the CC BY-NC-ND license (http://creativecommons.org/licenses/by-nc-nd/4.0/)
Acoustic signals support lower data rate compared with the electromagnetic signals, hence UWSNs have low data rate compared with terrestrial sensor networks. UWSN has limited energy, less storage and low bandwidth, hence its real challenge to develop efficient Routing protocol for UAC which should increase packet delivery ratio, reduce energy consumption and delay. Several researchers proposed several routing protocols for UWSN. However it could be enhanced for better performance. In this paper, Itinerary aware routing protocol is proposed to increase data delivery and more energy efficiency and reduced delay. In this paper, section II describes related works carried in data delivery of UWSN. Section III explains proposed Itinerary aware routing protocol. Section IV, draws the simulation results and section $\mathrm{V}$ describes conclusion of the paper.

\section{RELATED WORKS}

The characteristics and the difference between Twodimensional architectures and three-dimensional architectures of underwater sensor networks are analyzed in [1]. They discussed about main challenges in under water network deployment and its efficiency. Multicarrier communication for high speed underwater acoustic communication was discussed in [2]. They utilized multicarrier communication for transfer of video and image, estimated Doppler shift and analyzed channel characteristics. Adaptive and efficient cost aware routing strategy is proposed for Multi hop wireless network by S.Lee et al[3] they proposed geographic routing with normalized advance (NADV) as link metric.. They also suggest higher delivery ratio with frequent packet losses in high noise environments. Void nodes create huge packet loss in UWSN, hence D. Chen et al [4] addressed issues occurred due to void nodes and compared the characteristics of existing void handling techniques using planar graph, flooding, cost, geometric, heuristic and hybrid etc. Z. Shi et al[5] analyzed mobile UWSN with high propagation delay and increased node mobility using three dimensional network topology, They proposed Void Avoidance protocol using vector calculus to avoid void problem occurring in routing of mobile UWSNs. J.-H. Cui et al[6] analyzed 4D monitoring of local underwater events, they have utilized pressure levels to route data to Sonobuoys, they utilized hydraulic pressure is a cost metric for finding shortest path. S. Oktug et al [7] analyzed localization techniques and open research issues in UWSN. C. Detweiler et al [8] increased network life and decreased the communication cost of UWSN using two way communication techniques, they have proposed acoustic communication for the nodes under water and radio communication for surface nodes. 
The routing algorithm which can predict mobility and location of underwater nodes was proposed by C. Bagtzoglou et al [9], which also support scalability. E. Cayirci et al[10] proposed an architecture for wireless sensor network to maximize coverage of underwater surveillance using three dimensional sensor deployment. J. Jaffe et al [11] analyzed sensor networks adapted for remote ocean exploration using human intervention and without human intervention.. K. Zhou Yu et al[12] analyzed localization of sensors using magnetometers which optimizes localization by minimizing error. P. D. Milica Stojanovic [13] analyzed the performance of acoustic channel using modified 3D mobility model which utilize the concept of lower current speeds with increased depth. R. W. L. Coutinho et al [14] proposed Depth controlled routing protocol for UWSN, they have utilized vertical movement ability of sensor nodes. F. Kuhn et al[15] proposed geometric routing protocol using benefits of greedy and face algorithms.

In 1980s, packet radio network utilized position of the node as metric of routing algorithm. In geographic routing location information of node and its destination using global positioning system (GPS). In this type of routing message is routed to destination with the location information of destination without having much knowledge on topology of the network. A. Boukerche et al [16] proposed adjustable topology by moving void nodes to new depth in mobile UWSN, they also utilized greedy opportunistic forwarding for improving packet delivery ratio. Cross layer based reinforcement learning protocol proposed in [17] increases network complexity and reduce network life time. Rodolfo W. L. Coutinho et al [18] proposed routing protocol for UWSNs with adjustable topology named Geographic and opportunistic routing with Depth Adjustment (GEDAR). It recovers and re-routes the packets over void nodes, GEDAR forwards packets to next hop using localization information.. GEDAR avoids unnecessary retransmission of packets by classifying nodes with different priority level based on location. The node having lower priority stops transmission whenever the same packet is transmitted by high priority nodes. However, GEDAR has high storage overhead, more energy consumption and more delay. In this paper, Itinerary aware routing protocol (IARP) is proposed to improve reliability of data transfer with reduced energy consumption.

\section{PROPOSED WORKS}

The proposed Itinerary Aware Routing Protocol improves reliable data transfer by avoiding void nodes. This algorithm determines void nodes and estimates alternate next hop reliable and reachable path to gateways through improved probabilistic estimation. The proposed IARP improves efficiency of data forwarding with reduced overheads and retransmission. In proposed system each node predicts and updates its location information by its initial coarse location and updating information from other nodes. It avoids frequent location information from Global positioning system, which avoids unnecessary overheads and energy consumption.. The same packet forwarding of multiple nodes to Sonobuoys are avoided by collaborative communication between the nodes which reduces repeated data transfer and increases network life time.. Fig 1 shows proposed Itinerary aware routing protocol architecture.

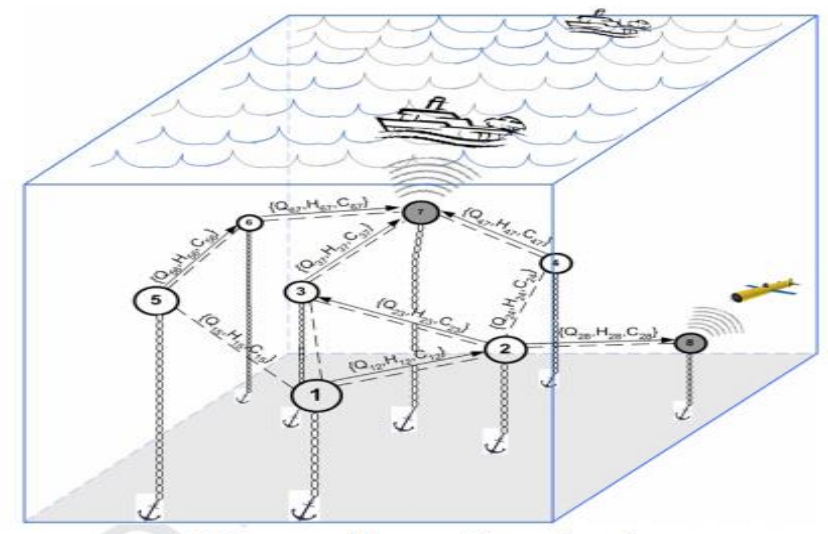

Fig .1 Architecture of Itinerary Aware Routing Protocol

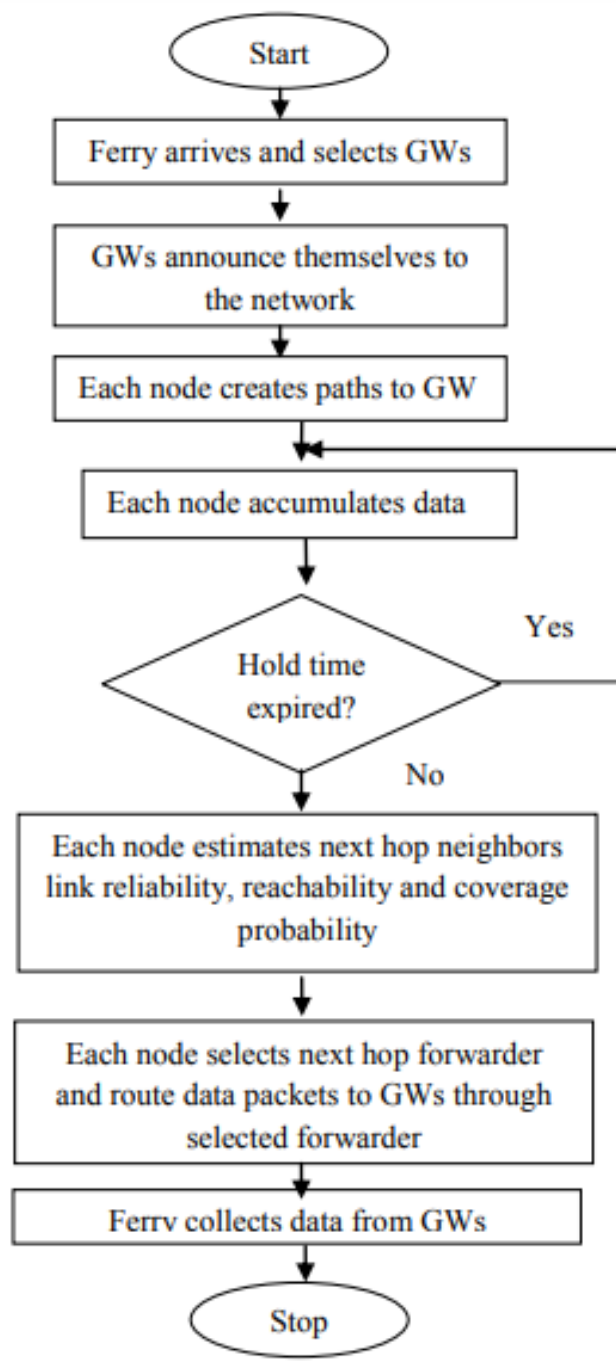

Fig 2. Flowchart of IARP

The proposed IARP considers mobility of nodes due to ocean current and other environmental forces acting on each node for predicting localization of each node which increases reliability of data transfer and improves robustness. The nodes having same information are not forwarded to sonobuoys which avoids duplicate data transfer and increases network life time. Fig 2 shows flow chart of proposed IARP. 
Link transmission reliability is estimated using equation(1),

$$
\hat{\mathrm{Q}}_{\mathrm{ij}}(\mathrm{t})=\left\{\begin{array}{cc}
\mathrm{a} \times \mathrm{Q}_{\mathrm{ij}}(\mathrm{t})+(1-\mathrm{a}) \times \hat{\mathrm{Q}}_{\mathrm{ij}}(\mathrm{t}-1) & \text { if } \mathrm{t}>1 \\
\mathrm{Q}_{\mathrm{ij}}(\mathrm{t}) & \text { if } \mathrm{t}=1
\end{array}\right.
$$

$\mathrm{Q}_{\mathrm{ij}}$ is probability of obtaining reliable communication between node $\mathrm{i}$ to $\mathrm{j}$

Reachability of next hop for forwarding is estimated using (2)

$$
P\left(H_{i j} \mid j\right)= \begin{cases}\frac{P\left(\hat{H}_{i j} \leq H_{i j}\right)}{H_{i j} \sum_{l=1}^{m} \frac{P\left(\hat{H}_{i l} \leq H_{i l}\right)}{H_{i l}}} & \text { if } t>1 \\ \frac{1}{H_{i j} \sum_{l=1}^{m} \frac{1}{H_{i l}}} & \text { if } t=1\end{cases}
$$

Coverage probability estimated using equation (3)

$$
P\left(C_{i j} \mid j\right)= \begin{cases}\frac{C_{i j} \times P\left(\hat{C}_{i j} \leq C_{i j}\right)}{\sum_{l=1}^{m} C_{i l} \times P\left(\hat{C}_{i l} \leq C_{i l}\right)} & \text { if } t>1 \\ \frac{C_{i j}}{\sum_{l=1}^{m} C_{i l}} & \text { if } t=1\end{cases}
$$

\section{SIMULATION RESULT AND DISCUSSION}

Simulation is used for the performance analysis of a created system model based on their functioning. Therefore, the assumption and estimation of a system can be achieved using the simulation results. The performance of the proposed IARP, existing DBR and GEDAR routing are verified using network stimulator NS2 software. The performance of proposed IARP is justified using energy consumption, end-to-end delay, throughput, Packet Delivery Ratio and Packet Loss. The parameters used for the simulation are given in the table .1

Table 1. Simulation Parameter

\begin{tabular}{|l|l|}
\hline Simulation tool & NS2 \\
\hline Software used & Linux/Windows XP \\
\hline Channel & Acoustic channel(property) \\
\hline Antenna & Omni directional antenna \\
\hline Maximum packet & 1024 \\
\hline Network interface type & Wireless physical layer \\
\hline Number of nodes & 40 \\
\hline X Co-ordinate & 1050 \\
\hline Y Co-ordinate & 850 \\
\hline Energy model & Coordinated energy model \\
\hline Initial energy in joules & 100 \\
\hline
\end{tabular}

\section{A. Energy Consumption and End-to-End Delay}

Fig. 3 shows the energy consumption of DBR, GEDAR and IARP. This shows that the consumption of energy decreases in IARP when compare to DBR and GEDAR.

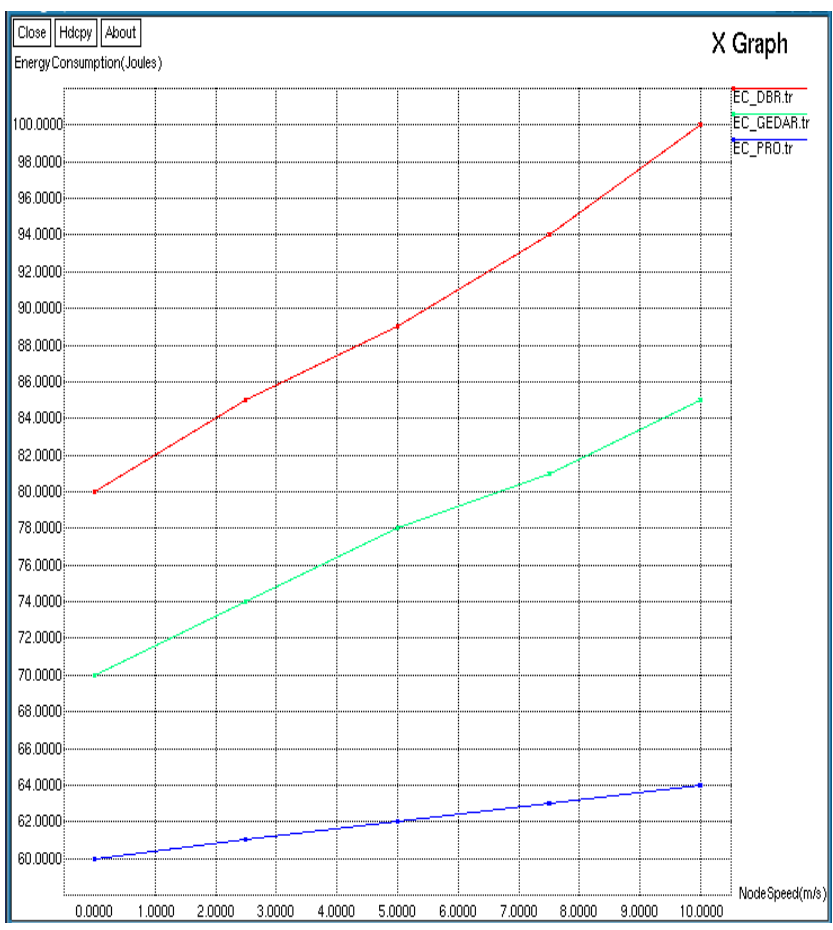

Fig 3. Energy consumption of DBR,GEDAR and IARP

Since the proposed IARP avoids unwanted transmission to void node effectively. The location information is estimated using reachability and coverage probability which reduces End- to- End delay compared with DBR and GEDAR as shown in Fig 4.

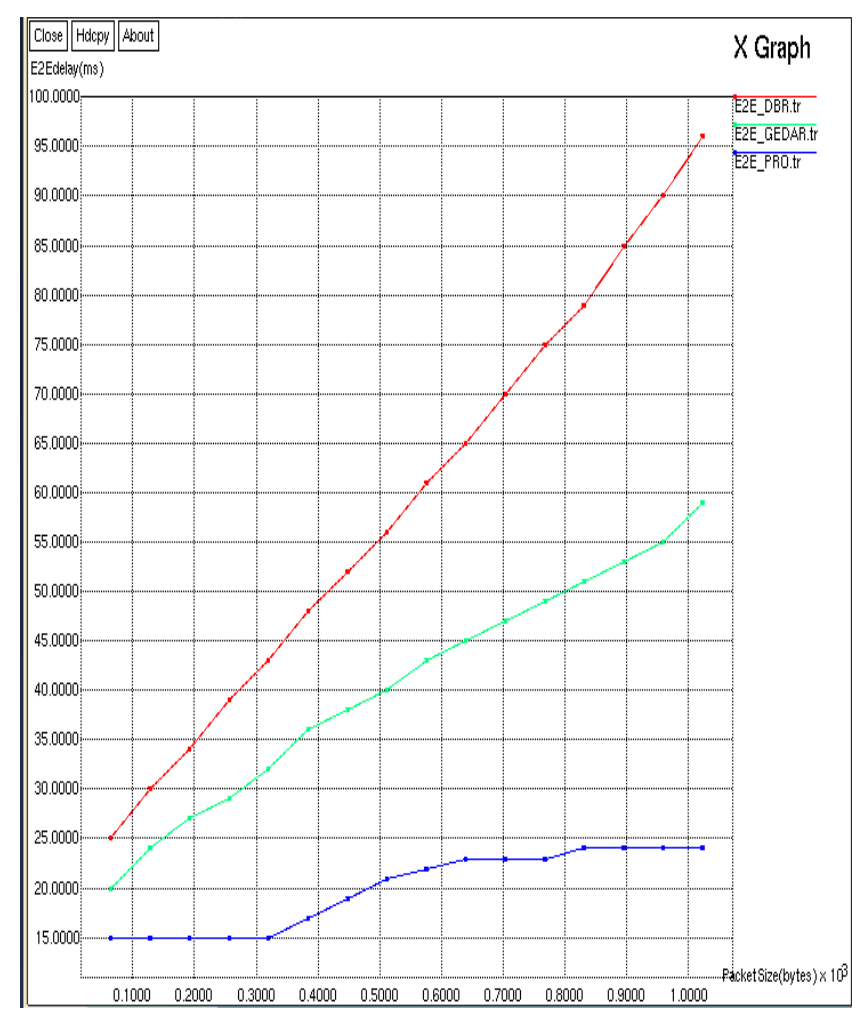

Fig 4. End- to-end delay of DBR,GEDAR and IARP

Published By:

Blue Eyes Intelligence Engineering 


\section{B. Throughput and packet Delivery ratio}

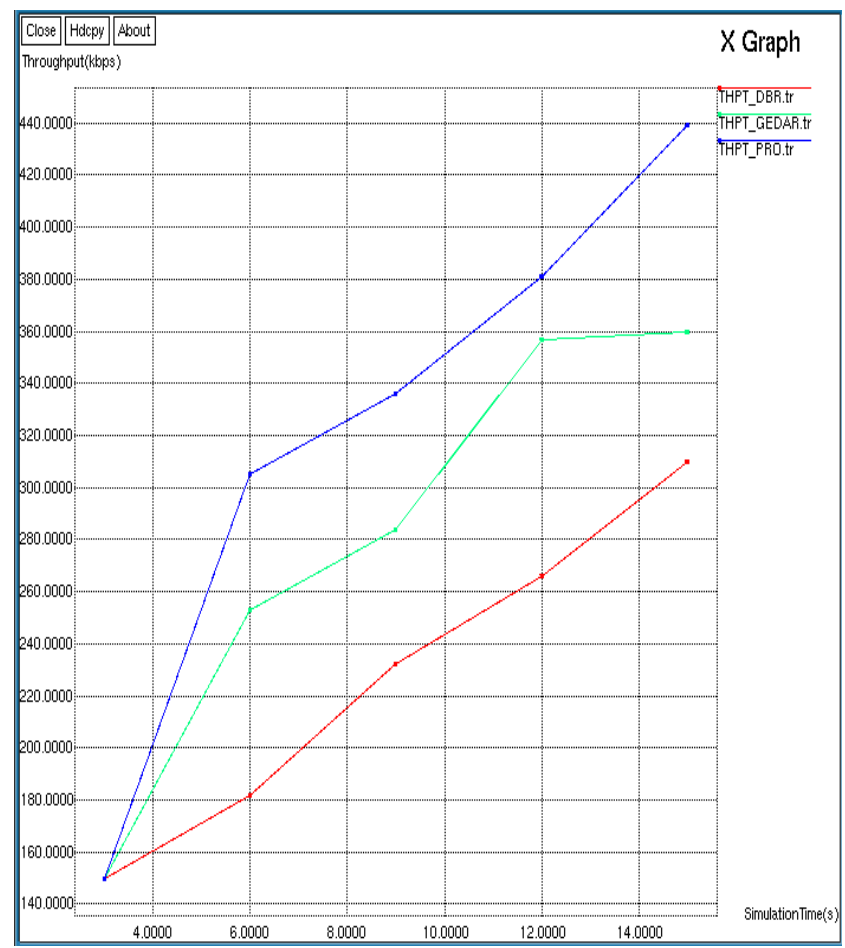

Fig 5. Throughput of DBR,GEDAR and IARP

Fig 5 and Fig 6 show the throughput and packet delivery ratio of DBR, GEDAR and IARP. During the transmission of beacon packet the throughput of IARP and packet delivery ratio are increased than the DBR and GEDAR since IARP estimates reliable path and avoids void nodes which increases throughput and packet delivery ratio.

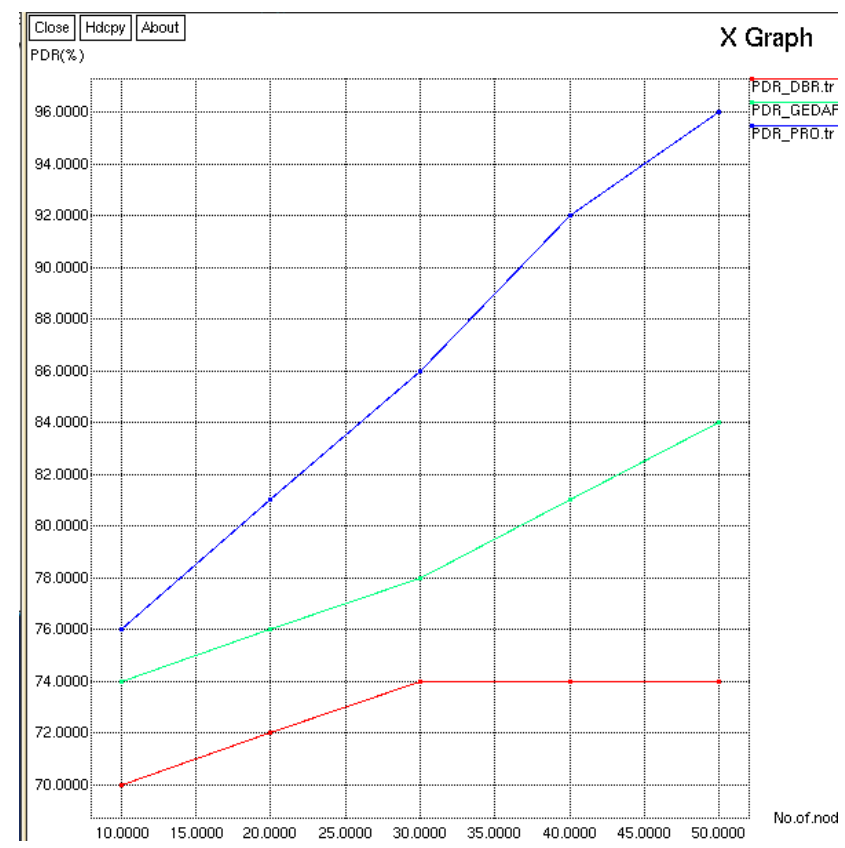

Fig.6. Packet delivery ratio of DBR,GEDAR and IARP

C. Routing Overhead and network lifetime

This Fig. 7 and 8 show the routing overhead and network life time of DBR,GEDAR and IARP. In DBR and GEDAR based routing location information of each node is transmitted to all node which increases routing overhead and decreases network life time. However, in IARP each node estimates its neighbor by reachability and probability hence the overhead get decreased and network life time increases.

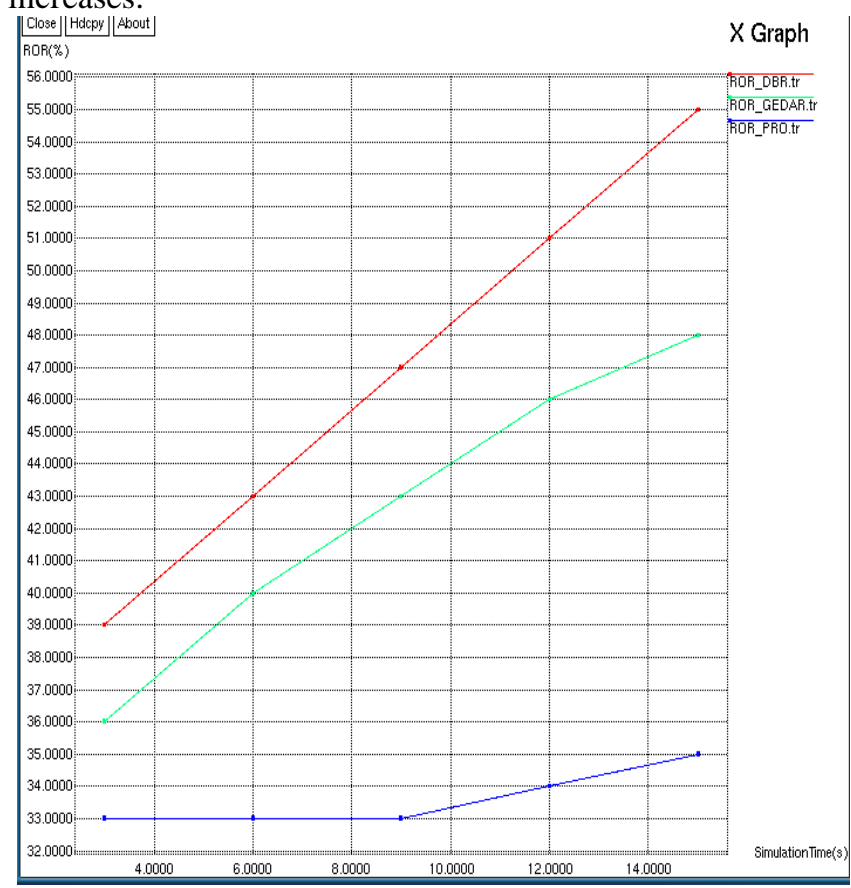

Fig 7. Routing overhead of DBR,GEDAR and IARP

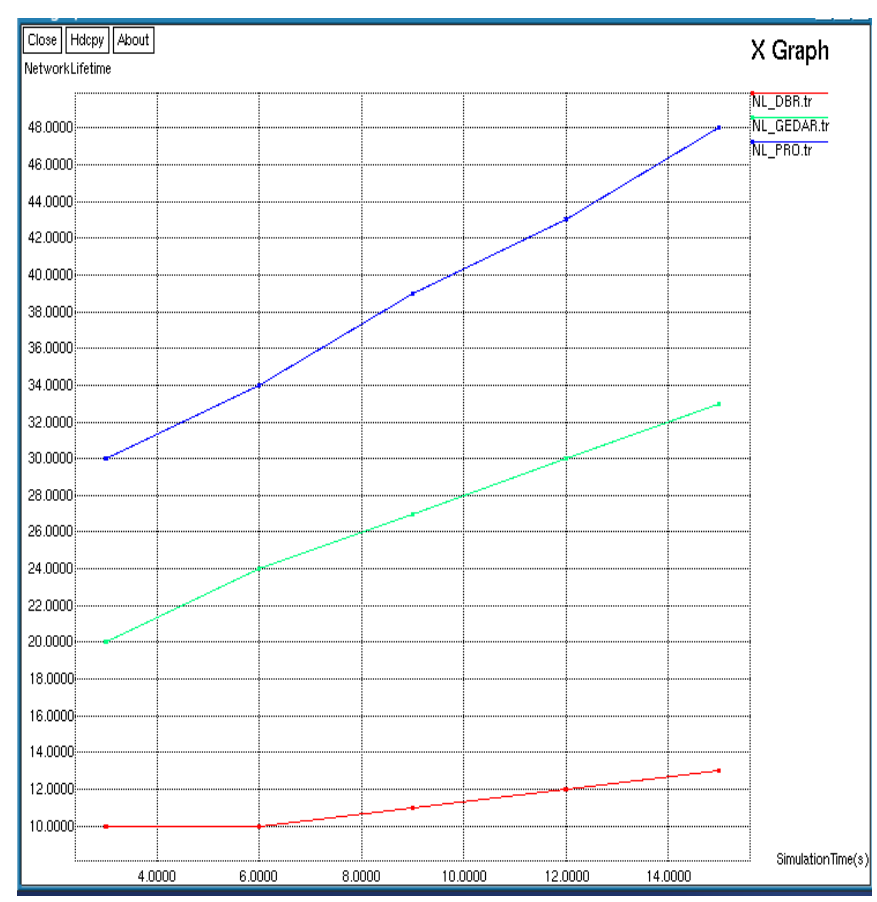

Fig 8. Network life time of DBR,GEDAR and IARP

\section{CONCLUSION}

The Itinerary aware routing protocol (IARP) is proposed to improve the reliable data delivery in under-water sensor networks and it is verified using simulation. IARP is a robust and scalable protocol which uses local information for predicting location and avoids redundancy in packet forwarding between the nodes and surface sonobuoys. Hence, required data transfer between sonobuoys and central station gets reduced which improves speed of data transfer .

Published By:

Blue Eyes Intelligence Engineering

DOI: 10.35940/ijeat.A1889.129219 
Our simulation results shows that itinerary aware routing protocols utilized efficient localization than the GEDAR routing protocols. Moreover, IARP improved the packet delivery ratio, throughput and the life time of network. IARP efficiently reduces the percentage of energy consumption when compare to GEDAR and depth adjustment routing.

\section{REFERENCES}

1. F. Akyildiz, D. Pompili, and T. Melodia, "Underwater acoustic sensor networks: Research challenges", Ad Hoc Network, Vol. 3, No.3, pp. 257-279, 2005.

2. Vasilescu, K. Kotay, D. Rus, M. Dunbabin, and P. Corke, "Data collection, storage, and retrieval with an underwater sensor network", Proceedings of ACM International Conference on Embedded Network Sensor System, pp. 154-165, 2005.

3. U. Lee, P. Wang, Y. Noh, L. F. M. Vieira, M. Gerla, and J.-H. Cui, "Pressure routing for underwater sensor networks", Proceedings of IEEE conference INFOCOM, pp. 1-9, 2010.

4. D. Chen and P. Varshney, "A survey of void handling techniques for geographic routing in wireless networks", IEEE Communication Surveys Tutorials, vol. 9, No. 1, pp. 50-67, 2007.

5. H. Yan, Z. J. Shi, and J.-H. Cui, "DBR: Depth-based routing for underwater sensor networks," Proceedings of Network Conference on Ad Hoc Sensor Network, Wireless Network, Next Generation Internet, pp. 72-86, 2008.

6. P. Xie, Z. Zhou, Z. Peng, J.-H. Cui, and Z. Shi, "Void avoidance in three-dimensional mobile underwater sensor networks," Proceedings of International Conference on Wireless Algorithms, System, Application, vol. 5682, pp. 305-314, 2009

7. M. Erol-Kantarci, H. Mouftah, and S. Oktug, "A survey of architectures and localization techniques for underwater acoustic sensor networks," IEEE Communication Surveys Tutorials, Vol.13, No. 3, pp. 487-502, 2011.

8. M. O'Rourke, E. Basha, and C. Detweiler, "Multi-modal communications in underwater sensor networks using depth adjustment", Proceeding of ACM International Conference on Underwater Network System, pp. 31:1-31:5, 2012

9. Z. Zhou, Z. Peng, J.-H. Cui, Z. Shi, andA. C. Bagtzoglou, "Scalable localization with mobility prediction for underwater sensor networks", IEEE Transactions on Mobile Computing, Vol. 10, No.3, pp. 335-348, 2011.

10. E. Cayirci, H. Tezcan, Y. Dogan, and V. Coskun, "Wireless sensor networks for underwater surveillance systems", Ad Hoc Networks, Vol. 4, No. 4, pp. 431-446, 2006.

11. J. Jaffe and C. Schurgers, "Sensor networks of freely drifting autonomous underwater explorers," Proceddings of ACM International Workshop on Underwater Network, pp. 93-96, 2006.

12. Zhou Yu, C. Xiao, and G. Zhou, "Multi-objectivization based localization of underwater sensors using magnetometers," IEEE Sensor Journal, Vol. 14, No.4, pp. 1099-1106, 2014.

13. Milica Stojanovic, "On the relationship between capacity and distance in an underwater acoustic communication channel", Proceedings of ACM International Workshop on Underwater Network, pp. 41-47, 2006.

14. R. W. L. Coutinho, L. F. M. Vieira, and A. A. F. Loureiro, "DCR Depth-controlled routing protocol for underwater sensor networks,' Proceedings of IEEE Symposium on Computer Communication, pp. 453-458., 2013

15. F. Kuhn, R. Wattenhofer, and A. Zollinger, "Worst-case optimal and average-case efficient geometric ad-hoc routing", Proceedings of ACM International Symposium on Mobile Ad Hoc Network, pp. 267-278, 2003.

16. Boukerche, L. F. M. Vieira, and A. A. Loureiro, "GEDAR Geographic and opportunistic routing protocol with depth adjustment for mobile underwater sensor. networks," Proc. IEEE International Conference on Communication, pp. 251-256, 2014.

17. Zhigang Jin, Qinyi Zhao, Yishan Su, "RCAR: A ReinforcementLearning-Based Routing Protocol for Congestion-Avoided Underwater Acoustic Sensor Networks", IEEE sensor journals, Early access, 2019.

18. Rodolfo W. L. Coutinho, Azzedine Boukerche, Luiz F. M. Vieira, and Antonio A. F. Loureiro, "Geographic and Opportunistic Routing for Underwater Sensor Networks" IEEE Transactions on computers, Vol. 65, No. 2, Feb 2016.

\section{AUTHORS PROFILE}

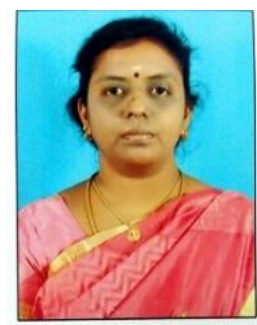

Dr. V. Bharathi Professor in Department of Electronics and Communication Engineering, Sri Manakula Vinayagar Engineering College. She had completed her Bachelor's degree in Engineering at 1999, Master of Engineering in 2003 and Ph.D from Pondicherry University in 2016. She has published 18 journals and 17 International. Her domains of interest are Wireless Networks, Wireless Communication and Signal Processing.

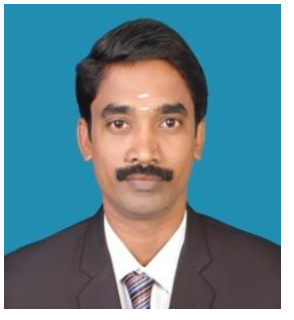

Dr. P. Raja is working as Professor in the Department of Electronics and Communication Engineering, Sri ManakulaVinayagar Engineering College. He received Bachelor of Engineering from Madras University, Master of Technology from IIT Madras and Doctor of Philosophy from Pondicherry University. He has two decades of teaching experience. His research are VLSI and Wireless Communication

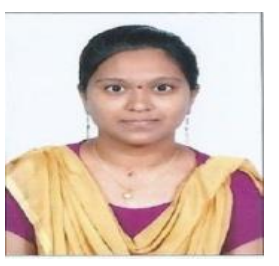

S. Meenabavyakarthika Completed B. Tech in Electronics and Communiation Engineering at Achariya College of Engineering Technology. Pursuing M.Tech in VLSI \& Embedded Systems at Sri Manakula Vinayagar Engineering College.

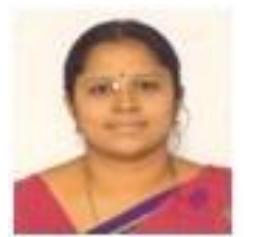

V. Logisvary working as Assistant Professor in Sri Manakula Vinayagar Engineering College in the department of ECE , I am having 8 years of teaching experience and completed my ME in Sathyabama University completed BE in VRS college of Engineering under Anna University

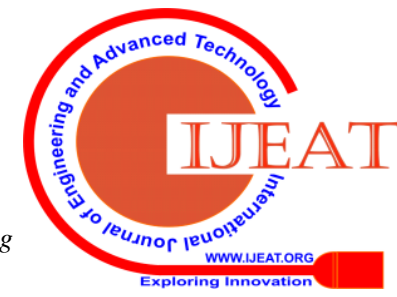

